\title{
Ambarzumian-type Problems for Discrete Schrödinger Operators
}

\author{
Burak Hatinoğlu ${ }^{1}$ (D) Jerik Eakins ${ }^{2}$. William Frendreiss ${ }^{2} \cdot$ Lucille Lamb $^{2}$. \\ Sithija Manage ${ }^{2}$. Alejandra Puente ${ }^{2}$
}

Received: 31 January 2021 / Accepted: 24 September 2021 / Published online: 16 October 2021

(C) The Author(s) 2021

\begin{abstract}
We discuss the problem of unique determination of the finite free discrete Schrödinger operator from its spectrum, also known as the Ambarzumian problem, with various boundary conditions, namely any real constant boundary condition at zero and Floquet boundary conditions of any angle. Then we prove the following Ambarzumian-type mixed inverse spectral problem: diagonal entries except the first and second ones and a set of two consecutive eigenvalues uniquely determine the finite free discrete Schrödinger operator.
\end{abstract}

Keyword Inverse spectral theory, Discrete Schrödinger operators, Ambarzumian-type problems

Communicated by Jussi Behrndt.

This article is part of the topical collection "Spectral Theory and Operators in Mathematical Physics" edited by Jussi Behrndt, Fabrizio Colombo and Sergey Naboko.

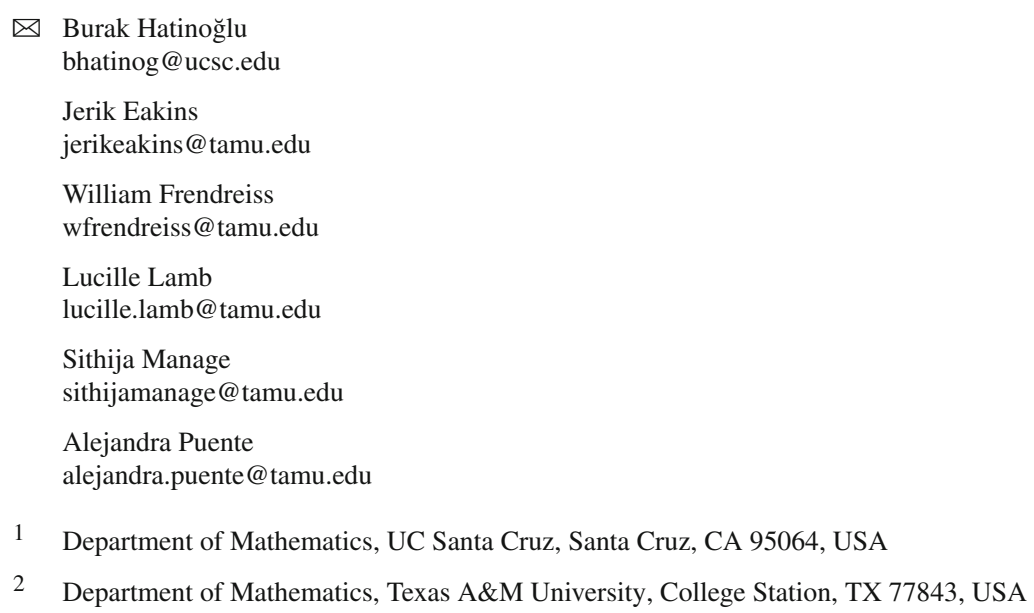




\section{Introduction}

The Jacobi matrix is a tridiagonal matrix defined as

$$
\left(\begin{array}{ccccc}
b_{1} & a_{1} & 0 & \ldots & 0 \\
a_{1} & b_{2} & a_{2} & \ddots & \vdots \\
0 & a_{2} & b_{3} & \ddots & 0 \\
\vdots & \ddots & \ddots & \ddots & a_{n-1} \\
0 & \ldots & 0 & a_{n-1} & b_{n}
\end{array}\right)
$$

where $n \in \mathbb{N}, a_{k}>0$ for any $k \in\{1,2, \ldots, n-1\}$ and $b_{k} \in \mathbb{R}$ for any $k \in$ $\{1,2, \ldots, n\}$. When $a_{k}=1$ for each $k \in\{1,2, \ldots, n-1\}$, the Jacobi matrix (1.1) defines the finite discrete Schrödinger operator.

Inverse spectral problems aim to recover $\left\{a_{k}\right\}_{k=1}^{n-1}$ and $\left\{b_{k}\right\}_{k=1}^{n}$ from spectral information. Ambarzumian-type problems focus on inverse spectral problems for free discrete Schrödinger operators, i.e. $a_{k}=1$ and $b_{k}=0$ for every $k$, or similar cases when $b_{k}=0$ for some $k$.

The study of inverse spectral problems of Schrödinger (Sturm-Liouville) equations goes back to Ambarzumian's work on a finite interval [1], and there are vast and still expanding literature on both continuous (see e.g. $[8,12,13,15,20]$ and references therein) and discrete (see e.g. [2-11,16,18] and references therein) settings.

In this paper, we first revisit the classical Ambarzumian problem for the finite discrete Schödinger operator in Theorem 3.3, which says that the spectrum of the free operator uniquely determines the operator. Then we provide a counterexample, Example 3.4, which shows that knowledge of the spectrum of the free operator with a nonzero boundary condition is not sufficient for unique recovery. In Theorem 3.5, we observe that a non-zero boundary condition along with the corresponding spectrum of the free operator is needed for the uniqueness result. However, in Theorem 3.6, we prove that for the free operator with Floquet boundary conditions, the set of eigenvalues including multiplicities is sufficient to get the uniqueness up to transpose.

We also answer the following mixed Ambarzumian-type inverse problem positively in Theorem 4.3.

Inverse Spectral Problem Let us consider the discrete Schrödinger matrix $\mathbf{S}_{n, 2}$ as $a_{k}=1$ for $k \in\{1, \ldots, n-1\}$ and $b_{1}, b_{2} \in \mathbb{R}, b_{k}=0$ for $k \in\{3, \ldots, n\}$. Let us also denote the free discrete Schrödinger operator by $\mathbf{F}_{n}$, which is defined as $a_{k}=1$ for $k \in\{1, \ldots, n-1\}$ and $b_{k}=0$ for $k \in\{1, \ldots, n\}$. If $\mathbf{S}_{n, 2}$ and $\mathbf{F}_{n}$ share two consecutive eigenvalues, then do we get $b_{1}=b_{2}=0$, i.e. $\mathbf{S}_{n, 2}=\mathbf{F}_{n}$ ?

The paper is organized as follows. In Sect. 2 we fix our notations. In Sect. 3 we consider the problem of unique determination of the finite free discrete Schrödinger operator from its spectrum, with various boundary conditions, namely any real constant boundary condition at zero, and Floquet boundary conditions of any angle. In Sect. 4 we prove the above mentioned Ambarzumian-type mixed inverse spectral problem. 


\section{Notations}

Let us start by fixing our notation. Let $\mathbf{S}_{n}$ represent the discrete Schrödinger matrix of size $n \times n$

$$
\mathbf{S}_{n}:=\left(\begin{array}{ccccc}
b_{1} & 1 & 0 & \cdots & 0 \\
1 & b_{2} & 1 & \ddots & \vdots \\
0 & 1 & b_{3} & \ddots & 0 \\
\vdots & \ddots & \ddots & \ddots & 1 \\
0 & \cdots & 0 & 1 & b_{n}
\end{array}\right)
$$

where $b_{k} \in \mathbb{R}$. Let $\mathbf{S}_{n}(b, B)$ denote the discrete Schrödinger matrix $\mathbf{S}_{n}$ satisfying $b_{1}=b$ and $b_{n}=B$. Let us also introduce the following matrices:

$$
\mathbf{S}_{n}(\theta):=\left(\begin{array}{ccccc}
b_{1} & 1 & 0 & \ldots & e^{2 \pi i \theta} \\
1 & b_{2} & 1 & \ddots & \vdots \\
0 & 1 & b_{3} & \ddots & 0 \\
\vdots & \ddots & \ddots & \ddots & 1 \\
e^{-2 \pi i \theta} & \ldots & 0 & 1 & b_{n}
\end{array}\right) \text { and } \mathbf{S}_{n, m}:=\left(\begin{array}{cccccc}
b_{1} & 1 & 0 & 0 & \ldots & 0 \\
1 & \ddots & 1 & 0 & \ddots & 0 \\
0 & 1 & b_{m} & 1 & \ddots & \vdots \\
0 & 0 & 1 & 0 & \ddots & 0 \\
\vdots & \ddots & \ddots & \ddots & \ddots & 1 \\
0 & \ldots & \ldots & 0 & 1 & 0
\end{array}\right)
$$

Let us denote the free discrete Schrödinger matrix of size $n \times n$ by $\mathbf{F}_{n}$ :

$$
\mathbf{F}_{n}:=\left(\begin{array}{ccccc}
0 & 1 & 0 & \cdots & 0 \\
1 & 0 & 1 & \ddots & \vdots \\
0 & 1 & 0 & \ddots & 0 \\
\vdots & \ddots & \ddots & \ddots & 1 \\
0 & \cdots & 0 & 1 & 0
\end{array}\right),
$$

so $\mathbf{F}_{n}(b, B)$ and $\mathbf{F}_{n}(\theta)$ are defined accordingly.

In addition, let $p_{n}(x)$ be the characteristic polynomial of $\mathbf{F}_{n}$ with zeroes $\lambda_{1}, \ldots, \lambda_{n}$ and let $q_{n}(x)$ be the characteristic polynomial of $\mathbf{S}_{n}$ with zeroes $\mu_{1}, \ldots, \mu_{n}$. The discrete Schrödinger matrix $\mathbf{S}_{n}$ has $n$ distinct simple eigenvalues, so we can order the eigenvalues as $\lambda_{1}<\lambda_{2}<\cdots<\lambda_{n}$ and $\mu_{1}<\mu_{2}<\cdots<\mu_{n}$. This property is valid for any Jacobi matrix and can be found in [19], which provides an extensive study of Jacobi operators.

Throughout the paper we will discuss our results via matrices using the notations introduced above, but let us note that the matrices $\mathbf{S}_{n}(b, B)$ and $\mathbf{S}_{n}(\theta)$ can be represented as difference equations. 
Remark 2.1 Given $\mathbf{S}_{n}$, let us consider the discrete Schrödinger matrix where all $b_{k}$ 's are the same as in $\mathbf{S}_{n}$ except $b_{1}$ and $b_{n}$ are replaced by $b_{1}+b$ and $b_{n}+B$ respectively for $b, B \in \mathbb{R}$, i.e.

$$
\mathbf{S}_{n}+b\left(\delta_{1}, \cdot\right) \delta_{1}+B\left(\delta_{n}, \cdot\right) \delta_{n}
$$

The discrete Schrödinger matrix (2.1) is given by the difference expression

$$
f_{k-1}+b_{k} f_{k}+f_{k+1}, \quad k \in\{1, \cdots, n\}
$$

with the boundary conditions

$$
f_{0}=b f_{1} \quad \text { and } \quad f_{n+1}=B f_{n} .
$$

In order to get a unique difference expression with these boundary conditions for a given discrete Schrödinger matrix, we can think of the first and the last diagonal entries of the matrix $\mathbf{S}_{n}$ as the boundary conditions at 0 and $n+1$ respectively. Therefore $\mathbf{S}_{n}(b, B)$ denotes the discrete Schrödinger matrix with boundary conditions $b$ at $0, B$ at $n+1$.

If we consider the difference expression (2.2) with the Floquet boundary conditions

$$
f_{0}=f_{n} e^{2 \pi i \theta} \quad \text { and } \quad f_{n+1}=f_{1} e^{-2 \pi i \theta}, \quad \theta \in[0, \pi)
$$

then we get the matrix representation $\mathbf{S}_{n}(\theta)$.

\section{Ambarzumian problem with various boundary conditions}

Firstly let us obtain the first three leading coefficients of $q_{n}(x)$. This is a well-known result, but we give a proof in order to make this section self-contained.

Lemma 3.1 The characteristic polynomial $q_{n}(x)$ of the discrete Schrödinger matrix $S_{n}$ has the form

$$
q_{n}(x)=x^{n}-\left(\sum_{i=1}^{n} b_{i}\right) x^{n-1}+\left(\sum_{1 \leq i<j \leq n} b_{i} b_{j}-(n-1)\right) x^{n-2}+Q_{n-2}(x),
$$

where $Q_{n-2}(x)$ is a polynomial of degree at most $n-2$.

Proof The characteristic polynomial $q_{n}(x)$ is given by $\operatorname{det}\left(x \mathbf{I}_{n}-\mathbf{S}_{n}\right)$. Let us consider Liebniz' formula for the determinants

$$
\operatorname{det}(\mathbf{A})=\sum_{\sigma \in \mathbb{S}_{n}} \operatorname{sgn}(\sigma) \prod_{i=1}^{n} \alpha_{i, \sigma(i)}
$$


where $\mathbf{A}=\left[\alpha_{i, j}\right]$ is an $n \times n$ matrix and sgn is the sign function of permutations in the permutation group $\mathbb{S}_{n}$. If we use (3.1) with the identity permutation, then $\operatorname{det}\left(x \mathbf{I}_{n}-\mathbf{S}_{n}\right)$ becomes $\prod_{i=1}^{n}\left(x-b_{i}\right)$, so we get that $q_{n}(x)$ is a monic polynomial and the coefficient of $x^{n-1}$ is $-\operatorname{tr}\left(\mathbf{S}_{n}\right)$.

The coefficient of the $x^{n-2}$ term of $q_{n}(x)$ is formed from the sum of all disjoint pairs of $a_{i}$. Hence we obtain $\sum_{1 \leq i<j \leq n} b_{i} b_{j}$.

The only other permutation that will yield an $x^{n-2}$ term is a transposition. However, if $|i-j| \geq 1$, then the product will be zero. Thus we are looking for transpositions where $|i-j|=1$. There are $n-1$ of these, namely $(1,2),(2,3), \ldots,(n-2, n-$ $1),(n-1, n)$. The product is of the form

$$
\prod_{i=1}^{n} \alpha_{i, \sigma(i)}=\frac{(-1)(-1)\left(x-b_{1}\right)\left(x-b_{2}\right) \cdots\left(x-b_{n}\right)}{\left(x-b_{i}\right)\left(x-b_{j}\right)}=x^{n-2}+r_{n-3}(x),
$$

where $r_{n-3}(x)$ is a polynomial of degree at most $n-3$. Since the signature of a transposition is negative, we derive $-x^{n-2}$ for each product. Summing over all $n-1$ permutations and adding to $\sum_{1 \leq i<j \leq n} b_{i} b_{j}$ yields our desired result.

Corollary 3.2 The characteristic polynomial $p_{n}(x)$ of the free discrete Schrödinger matrix $\boldsymbol{F}_{n}$ has the form

$$
p_{n}(x)=x^{n}-(n-1) x^{n-2}+P_{n-2}(x),
$$

where $P_{n-2}(x)$ is a polynomial of degree at most $n-2$.

Proof Simply set $b_{i}=0$ for each $i \in\{1, \cdots, n\}$ and apply Lemma 3.1.

Now let us give a proof of Ambarzumian problem with Dirichlet-Dirichlet boundary conditions, i.e. for the matrix $\mathbf{F}_{n}(0,0)=\mathbf{F}_{n}$ in our notation.

Theorem 3.3 Suppose $\boldsymbol{S}_{n}$ shares all of its eigenvalues with $\boldsymbol{F}_{n}$. Then $\boldsymbol{S}_{n}=\boldsymbol{F}_{n}$.

Proof In order for the two matrices to have all the same eigenvalues, they must have equal characteristic polynomials. Comparing the results from Lemma 3.1 to Corollary 3.2 , we must have

$$
\sum_{i=1}^{n} b_{i}=0 \quad \text { and } \quad \sum_{1 \leq i<j \leq n} b_{i} b_{j}=0
$$

This leads us to conclude that

$$
\sum_{i=1}^{n} b_{i}^{2}=\left(\sum_{i=1}^{n} b_{i}\right)^{2}-2\left(\sum_{1 \leq i<j \leq n} b_{i} b_{j}\right)=0
$$

which only occurs when all the $b_{i}$ are zero, i.e. $\mathbf{S}_{n}=\mathbf{F}_{n}$, since $b_{i} \in \mathbb{R}$ for each $i \in\{1, \cdots, n\}$. 
A natural question to ask is whether or not we get the uniqueness of the free operator with non-zero boundary conditions. After Theorem 3.3, one may expect to get the uniqueness of a free discrete Schrödinger operator from a spectrum with nonzero boundary condition at 0 . However, this is not the case because of the following counterexample:

Example 3.4 Let us define the discrete Schrödinger matrices

$$
A:=\left(\begin{array}{lll}
2 & 1 & 0 \\
1 & 0 & 1 \\
0 & 1 & 0
\end{array}\right) \text { and } B:=\left(\begin{array}{ccc}
-2 /(1+\sqrt{5}) & 1 & 0 \\
1 & 1 & 1 \\
0 & 1(1+\sqrt{5}) / 2
\end{array}\right)
$$

The matrices $A$ and $B$ have the same characteristic polynomial $x^{3}-2 x^{2}-2 x+2$, so they share the same spectrum.

This example shows that Theorem 3.3 is a special case, so in order to get the uniqueness of a rank-one perturbation of the free operator, we also need to know the non-zero boundary condition along with the spectrum.

Theorem 3.5 Suppose $\boldsymbol{S}_{n}\left(b, b_{n}\right)$ shares all of its eigenvalues with $\boldsymbol{F}_{n}(b, 0)$. Then $S_{n}\left(b, b_{n}\right)=\boldsymbol{F}_{n}(b, 0)$.

Proof Comparing coefficients of characteristic polynomials of $\mathbf{S}_{n}\left(b, b_{n}\right)$ and $\mathbf{F}_{n}(b, 0)$ like we did in the proof of Theorem 3.3, we get

$$
b+\sum_{i=2}^{n} b_{i}=b \quad \text { and } \quad b \sum_{j=2}^{n} b_{j}+\sum_{2 \leq i<j \leq n} b_{i} b_{j}=0
$$

The first equation of (3.3) gives $\sum_{i=2}^{n} b_{i}=0$ and using this in the second equation of (3.3) we get $\sum_{2 \leq i<j \leq n} b_{i} b_{j}=0$. This leads us to conclude that

$$
\sum_{i=2}^{n} b_{i}^{2}=\left(\sum_{i=2}^{n} b_{i}\right)^{2}-2\left(\sum_{2 \leq i<j \leq n} b_{i} b_{j}\right)=0
$$

which only occurs when $b_{i}=0$ for each $i \in\{2, \cdots, n\}$, i.e. $\mathbf{S}_{n}\left(b, b_{n}\right)=\mathbf{F}_{n}(b, 0)$.

Now, we approach the Ambarzumian problem with Floquet boundary conditions. Let us recall that $\mathbf{S}_{n}(\theta)$ and $\mathbf{F}_{n}(\phi)$ denote a discrete Schrödinger operator and the free discrete Schrödinger operator with Floquet boundary conditions for the angles $0 \leq \theta<1$ and $0 \leq \phi<1$, respectively.

The following theorem shows that with Floquet boundary conditions, the knowledge of the spectrum of the free operator is sufficient for the uniqueness up to transpose.

Theorem 3.6 Suppose that $\boldsymbol{S}_{n}(\theta)$ shares all of its eigenvalues with $\boldsymbol{F}_{n}(\phi)$, including multiplicity, for $0 \leq \theta, \phi<1$. Then $b_{1}=\cdots=b_{n}=0$ and $\theta \in\{\phi, 1-\phi\}$, i.e. $S_{n}(\theta)$ $=\boldsymbol{F}_{n}(\phi)$ or $\boldsymbol{F}_{n}^{T}(\phi)$ 
Proof Let us define $D[k, l]$ as the following determinant of a $(l-k+1) \times(l-k+1)$ matrix for $1 \leq k<l \leq n$ :

$$
D[k, l]:=\left|\begin{array}{ccccc}
x-b_{k} & -1 & 0 & \cdots & 0 \\
-1 & x-b_{k+1} & \ddots & \ddots & \vdots \\
0 & \ddots & \ddots & \ddots & 0 \\
\vdots & \ddots & \ddots & x-b_{l-1} & -1 \\
0 & \cdots & 0 & -1 & x-b_{l}
\end{array}\right|_{(l-k+1)}
$$

Let us consider the characteristic polynomial of $\mathbf{S}_{n}(\theta)$ by using cofactor expansion on the first row:

$$
\begin{aligned}
& \left|x \mathbf{I}_{n}-\mathbf{S}_{n}(\theta)\right|=\left(x-b_{1}\right) D[2, n]+\left|\begin{array}{ccccc}
-1 & -1 & 0 & \cdots & 0 \\
-1 & x-b_{3} & -1 & \ddots & \vdots \\
0 & -1 & \ddots & \ddots & 0 \\
\vdots & \ddots & \ddots & x-b_{n-1} & -1 \\
-e^{-2 \pi i \theta} & 0 & \cdots & -1 & x-b_{n}
\end{array}\right|_{(n-1)} \\
& +(-1)^{n+1}\left(-e^{2 \pi i \theta}\right)\left|\begin{array}{ccccc}
-1 & x-b_{2} & -1 & 0 & \cdots \\
0 & -1 & x-b_{3} & \ddots & \ddots \\
\vdots & \ddots & \ddots & \ddots & -1 \\
0 & \cdots & 0 & -1 & x-b_{n-1} \\
-e^{-2 \pi i \theta} & 0 & \cdots & 0 & -1
\end{array}\right|_{(n-1)}
\end{aligned}
$$

Then by using cofactor expansions on the first row of the determinant in the second term and on the first column of the determinant in the third term we get

$$
\begin{aligned}
\left|x \mathbf{I}_{n}-\mathbf{S}_{n}(\theta)\right|=\left(x-b_{1}\right) D[2, n]+(-1) D[3, n]+\left|\begin{array}{ccccc}
0 & -1 & 0 & \cdots & 0 \\
0 & x-b_{4} & -1 & \ddots & \vdots \\
0 & -1 & \ddots & \ddots & 0 \\
\vdots & \ddots & \ddots & \ddots & -1 \\
-e^{-2 \pi i \theta} & 0 & \cdots & -1 & x-b_{n}
\end{array}\right|_{(n-2)} \\
\\
+(-1)^{n+1}\left(-e^{2 \pi i \theta}\right)(-1)\left|\begin{array}{ccccc}
-1 & x-b_{3} & -1 & 0 & \cdots \\
0 & -1 & x-b_{4} & \ddots & \ddots \\
\vdots & \ddots & \ddots & \ddots & -1 \\
0 & \cdots & \ddots & -1 & x-b_{n-1} \\
0 & \cdots & \cdots & 0 & -1
\end{array}\right|_{(n-2)} \\
+(-1)^{n+1}\left(-e^{2 \pi i \theta}\right)(-1)^{n}\left(-e^{-2 \pi i \theta}\right) D[2, n-1]
\end{aligned}
$$


Now let's use cofactor expansion on the first column of the determinant in the third term. We see that the determinant in the fourth term is the determinant of an upper triangular matrix. Therefore,

$$
\begin{aligned}
\left|x \mathbf{I}_{n}-\mathbf{S}_{n}(\theta)\right|= & \left(x-b_{1}\right) D[2, n]-D[3, n] \\
& +(-1)^{n-1}\left(-e^{2 \pi i \theta}\right)\left|\begin{array}{ccccc}
-1 & 0 & 0 & \cdots & 0 \\
x-b_{4} & -1 & \ddots & \ddots & \vdots \\
-1 & x-b_{5} & \ddots & \ddots & 0 \\
0 & \ddots & \ddots & -1 & 0 \\
-e^{-2 \pi i \theta} & 0 & -1 & x-b_{n-1} & -1
\end{array}\right|_{(n-3)} \\
& +(-1)^{n+1}\left(-e^{2 \pi i \theta}\right)\left[(-1)(-1)^{n-2}+(-1)^{n}\left(-e^{-2 \pi i \theta}\right) D[2, n-1]\right]
\end{aligned}
$$

Finally, observing that the determinant in the third term is that of a lower triangular matrix, we get

$$
\begin{aligned}
\left|x \mathbf{I}_{n}-\mathbf{S}_{n}(\theta)\right|= & \left(x-b_{1}\right) D[2, n]-D[3, n]+(-1)^{n-1}\left(-e^{2 \pi i \theta}\right)(-1)^{n-3} \\
& +(-1)^{2 n}\left(-e^{-2 \pi i \theta}\right)+(-1)^{2 n+1} D[2, n-1] \\
& =\left(x-b_{1}\right) D[2, n]-D[3, n]-D[2, n-1]-e^{2 \pi i \theta}-e^{-2 \pi i \theta}
\end{aligned}
$$

At this point note that $D[k, l]$ is the characteristic polynomial of the following discrete Schrödinger matrix

$$
\left(\begin{array}{ccccc}
b_{k} & 1 & 0 & \cdots & 0 \\
1 & b_{k+1} & 1 & \ddots & \vdots \\
0 & 1 & \ddots & \ddots & 0 \\
\vdots & \ddots & \ddots & b_{l-1} & 1 \\
0 & \cdots & 0 & 1 & b_{l}
\end{array}\right) .
$$

Therefore using Lemma 3.1 and Eq. (3.4), we obtain

$$
\begin{aligned}
\left|x \mathbf{I}_{n}-\mathbf{S}_{n}(\theta)\right|= & x^{n}-\left(\sum_{i=1}^{n} b_{i}\right) x^{n-1}+\left(\sum_{1 \leq i<j \leq n} b_{i} b_{j}-(n-1)\right) x^{n-2} \\
& +f_{n-3}(x)-e^{2 \pi i \theta}-e^{-2 \pi i \theta}
\end{aligned}
$$

where $f_{n-3}$ is a polynomial of degree at most $n-3$, independent of $\theta$. Using the same steps for $\mathbf{F}_{n}(\phi)$, we obtain

$$
\left|x \mathbf{I}_{n}-\mathbf{F}_{n}(\phi)\right|=x^{n}-(n-1) x^{n-2}+g_{n-3}(x)-e^{2 \pi i \phi}-e^{-2 \pi i \phi}
$$


where $g_{n-3}$ is a polynomial of degree at most $n-3$, which is independent of $\phi$.

Comparing Eqs. (3.5) and (3.6), like we did in the Proof of Theorem 3.3, we can conclude that the diagonal entries $\left\{b_{i}\right\}_{i=1}^{n}$ of $\mathbf{S}_{n}(\theta)$ must be zero.

Note that the expression consisting of the first three terms in the right end of (3.4), $\left(x-b_{1}\right) D[2, n]-D[3, n]-D[2, n-1]$ is independent of $\theta$. In addition, we observed that $b_{1}=\cdots=b_{n}=0$. Therefore using the equivalence of the characteristic polynomials of $\mathbf{S}_{n}(\theta)$ and $\mathbf{F}_{n}(\phi)$, we obtain

$$
e^{2 \pi i \theta}+e^{-2 \pi i \theta}=e^{2 \pi i \phi}+e^{-2 \pi i \phi},
$$

which can be written using Euler's identity as

$$
2 \cos (2 \pi \theta)=2 \cos (2 \pi \phi) .
$$

Equation (3.7) is valid if and only if $\theta$ differs from $\phi$ or $-\phi$ by an integer. Since $0 \leq \theta, \phi<1$, the only possible values for $\theta$ are $\phi$ and $1-\phi$. This completes the proof.

\section{An Ambarzumian-type mixed inverse spectral problem}

Let us recall that $\mathbf{S}_{n, m}$ denotes the following $n \times n$ discrete Schrödinger matrix for $1 \leq m \leq n$ :

$$
\mathbf{S}_{n, m}:=\left(\begin{array}{cccccc}
b_{1} & 1 & 0 & 0 & \ldots & 0 \\
1 & \ddots & 1 & 0 & \ddots & 0 \\
0 & 1 & b_{m} & 1 & \ddots & \vdots \\
0 & 0 & 1 & 0 & \ddots & 0 \\
\vdots & \ddots & \ddots & \ddots & \ddots & 1 \\
0 & \ldots & \ldots & 0 & 1 & 0
\end{array}\right)
$$

and $\mathbf{F}_{n}$ denotes the free discrete Schrödinger matrix of size $n \times n$. In this section our goal is to answer the following Ambarzumian-type mixed spectral problem positively for the $m=2$ case.

Inverse Spectral Problem If $\mathbf{S}_{n, m}$ and $\mathbf{F}_{n}$ share $m$ consecutive eigenvalues, then do we get $b_{1}=\cdots=b_{m}=0$, i.e. $\mathbf{S}_{n, m}=\mathbf{F}_{n}$ ?

When $m=1$, this problem becomes a special case of the following result of Gesztesy and Simon [10]. For a Jacobi matrix given as (1.1), let us consider the sequences $\left\{a_{k}\right\}$ and $\left\{b_{k}\right\}$ as a single sequence $b_{1}, a_{1}, b_{2}, a_{2}, \cdots, a_{n-1}, b_{n}=$ $c_{1}, c_{2}, \cdots, c_{2 n-1}$, i.e. $c_{2 k-1}:=b_{k}$ and $c_{2 k}:=a_{k}$.

Theorem 4.1 ([10], Theorem 4.2) Suppose that $1 \leq k \leq n$ and $c_{k+1}, \cdots, c_{2 n-1}$ are known, as well as $k$ of the eigenvalues. Then $c_{1}, \cdots, c_{k}$ are uniquely determined. 
Proposition 4.2 Let $\lambda_{k}=\tilde{\lambda}_{k}$ for some $k \in\{1,2, \ldots, n-1\}$. Then $b_{1}=0$, i.e. $\boldsymbol{S}_{n, 1}=\boldsymbol{F}_{n}$.

Proof Following the notations of Theorem 4.1, $c_{2}, \cdots, c_{2 n-1}$ are known for $\mathbf{S}_{n, 1}$. Hence by letting $k=1$ in Theorem 4.1 , we get $\mathbf{S}_{n, 1}=\mathbf{F}_{n}$.

Now let us prove the $m=2$ case. Let $\lambda_{1}<\lambda_{2}<\cdots<\lambda_{n}$ denote the eigenvalues of $\mathbf{F}_{n}$, and let $\tilde{\lambda}_{1}<\tilde{\lambda}_{2}<\cdots<\tilde{\lambda}_{n}$ denote the eigenvalues of $\mathbf{S}_{n, 2}$.

Theorem 4.3 Let $\lambda_{k}=\tilde{\lambda}_{k}$ and $\lambda_{k+1}=\tilde{\lambda}_{k+1}$ for some $k \in\{1,2, \ldots, n-1\}$. Then $b_{1}=0$ and $b_{2}=0$, i.e. $\boldsymbol{S}_{n, 2}=\boldsymbol{F}_{n}$.

Proof We start by proving the following claim.

Claim: If $\lambda_{k}=\tilde{\lambda}_{k}$ and $\lambda_{k+1}=\tilde{\lambda}_{k+1}$, then either $b_{1}=b_{2}=0$ or $b_{1}=\lambda_{k}+\lambda_{k+1}$ and $b_{2}=1 / \lambda_{k}+1 / \lambda_{k+1}$.

Let us consider the characteristic polynomial of $\mathbf{S}_{n, 2}$ using cofactor expansion on the first row of $\lambda I-\mathbf{S}_{n, 2}$.

$$
\begin{aligned}
& \operatorname{det}\left(\lambda I-\mathbf{S}_{n, 2}\right)= \\
& \left(\lambda-b_{1}\right)\left|\begin{array}{ccccc}
\lambda-b_{2} & -1 & 0 & \ldots & 0 \\
-1 & \lambda & -1 & \ddots & \vdots \\
0 & -1 & \lambda & \ddots & 0 \\
\vdots & \ddots & \ddots & \ddots & -1 \\
0 & \ldots & 0 & -1 & \lambda
\end{array}\right|_{(n-1)}+\left|\begin{array}{ccccc}
-1 & -1 & 0 & \ldots & 0 \\
-1 & \lambda & -1 & \ddots & \vdots \\
0 & -1 & \lambda & \ddots & 0 \\
\vdots & \ddots & \ddots & \ddots & -1 \\
0 & \ldots & 0 & -1 & \lambda
\end{array}\right|_{(n-1)}
\end{aligned}
$$

Using cofactor expansion on the first row for the first term and the first column for the second term, we get

$$
\begin{aligned}
\operatorname{det}\left(\lambda I-\mathbf{S}_{n, 2}\right)= & \left(\lambda-b_{1}\right)\left(\lambda-b_{2}\right) \operatorname{det}\left(\lambda I-\mathbf{F}_{n-2}\right) \\
& +\left(\lambda-b_{1}\right)\left|\begin{array}{ccccc}
-1 & -1 & 0 & \ldots & 0 \\
0 & \lambda & -1 & \ddots & \vdots \\
0 & -1 & \lambda & \ddots & 0 \\
\vdots & \ddots & \ddots & \ddots & -1 \\
0 & \ldots & 0 & -1 & \lambda
\end{array}\right|_{(n-2)}-\operatorname{det}\left(\lambda I-\mathbf{F}_{n-2}\right)
\end{aligned}
$$

Finally, using cofactor expansion on the first column of the second term, we get

$$
\begin{aligned}
& \operatorname{det}\left(\lambda I-\mathbf{S}_{n, 2}\right)=\left[\left(\lambda-b_{1}\right)\left(\lambda-b_{2}\right)-1\right] \operatorname{det}\left(\lambda I-\mathbf{F}_{n-2}\right) \\
& -\left(\lambda-b_{1}\right) \operatorname{det}\left(\lambda I-\mathbf{F}_{n-3}\right) .
\end{aligned}
$$

Note that the cofactor expansions we derived so far are given in [10] in a more general setting for Jacobi matrices. One just needs to look at (2.4) in [10] and keep in mind that $(2.5)$ in $[10]$ takes place. 
Since $\tilde{\lambda}_{k}=\lambda_{k}$ and $\tilde{\lambda}_{k+1}=\lambda_{k+1}$, right hand side of (4.1) is zero when $\lambda=\lambda_{k}$ or $\lambda=\lambda_{k+1}$. Therefore for $\lambda=\lambda_{k}$ or $\lambda=\lambda_{k+1}$ we get

$$
\frac{\left(\lambda-b_{1}\right)\left(\lambda-b_{2}\right)-1}{\lambda-b_{1}}=\frac{\operatorname{det}\left(\lambda I-\mathbf{F}_{n-3}\right)}{\operatorname{det}\left(\lambda I-\mathbf{F}_{n-2}\right)} .
$$

Equation (4.2) is also valid for $\mathbf{F}_{n}$, i.e. when $b_{1}=b_{2}=0$, and the right hand side of the equation does not depend on $b_{1}$ or $b_{2}$ and hence identical for $\mathbf{S}_{n, 2}$ and $\mathbf{F}_{n}$. Therefore the left hand side of (4.2) should also be identical for $\mathbf{S}_{n, 2}$ and $\mathbf{F}_{n}$, when $\tilde{\lambda}_{k}=\lambda_{k}$ and $\tilde{\lambda}_{k+1}=\lambda_{k+1}$. Hence,

$$
\frac{\left(\lambda-b_{1}\right)\left(\lambda-b_{2}\right)-1}{\lambda-b_{1}}=\frac{(\lambda-0)(\lambda-0)-1}{\lambda-0}
$$

for $\lambda=\lambda_{k}$ or $\lambda=\lambda_{k+1}$. Therefore,

$$
\begin{aligned}
\lambda\left(\lambda-b_{1}\right)\left(\lambda-b_{2}\right)-\lambda & =\left(\lambda^{2}-1\right)\left(\lambda-b_{1}\right) \\
\lambda^{3}-\left(b_{1}+b_{2}\right) \lambda^{2}+b_{1} b_{2} \lambda-\lambda & =\lambda^{3}-b_{1} \lambda^{2}-\lambda+b_{1} \\
-b_{2} \lambda^{2}+b_{1} b_{2} \lambda-b_{1} & =0
\end{aligned}
$$

for $\lambda=\lambda_{1}$ or $\lambda=\lambda_{2}$. If $b_{2}=0$, then $b_{1}=0$ from the last equation above, so we can assume $b_{2} \neq 0$. Then $\lambda^{2}-b_{1} \lambda+b_{1} / b_{2}=0$ for $\lambda=\lambda_{k}$ or $\lambda=\lambda_{k+1}$.

Since $x^{2}-b_{1} x+b_{1} / b_{2}$ is a monic polynomial with two distinct roots $x=\lambda_{k}$ and $x=\lambda_{k+1}$, we get

$$
x^{2}-b_{1} x+b_{1} / b_{2}=\left(x-\lambda_{k}\right)\left(x-\lambda_{k+1}\right)
$$

which implies

$$
x^{2}-b_{1} x+b_{1} / b_{2}=x-\left(\lambda_{k}+\lambda_{k+1}\right) x+\lambda_{k} \lambda_{k+1}
$$

Comparing coefficients we get our claim, since $b_{1}=\lambda_{k}+\lambda_{k+1}$, and $b_{1} / b_{2}=\lambda_{k} \lambda_{k+1}$ implies

$$
b_{2}=\frac{b_{1}}{\lambda_{k} \lambda_{k+1}}=\frac{\lambda_{k}+\lambda_{k+1}}{\lambda_{k} \lambda_{k+1}}=\frac{1}{\lambda_{k}}+\frac{1}{\lambda_{k+1}}
$$

Now our goal is to get a contradiction for the second case of the claim, i.e. when $b_{1}=\lambda_{k}+\lambda_{k+1}$ and $b_{2}=1 / \lambda_{k}+1 / \lambda_{k+1}$, so let us assume

$$
b_{1}=\lambda_{k}+\lambda_{k+1} \quad \text { and } \quad b_{2}=1 / \lambda_{k}+1 / \lambda_{k+1} .
$$

First let us show that $b_{1}$ and $b_{2}$ have the same sign. If $n$ is even and $k=n / 2$, then $\lambda_{k}=-\lambda_{k+1}$. Hence $b_{1}=b_{2}=0$. If $n$ is odd and $k=(n-1) / 2$ or $k=(n+1) / 2$, then one of the eigenvalues $\lambda_{k}$ or $\lambda_{k+1}$ is zero, so $b_{2}$ is undefined. For all other values 
of $k$, two consecutive eigenvalues $\lambda_{k}$ and $\lambda_{k+1}$ and hence $b_{1}$ and $b_{2}$ have the same sign.

Without loss of generality, let us assume both $\lambda_{k}$ and $\lambda_{k+1}$ are negative and $b_{1} \leq b_{2}$. Let us define the matrix $\mathbf{M}_{n}(t)$ with the real parameter $t$ as follows:

$$
\mathbf{M}_{n}(t):=\left(\begin{array}{ccccc}
-t & 1 & 0 & \ldots & 0 \\
1 & -t & 1 & \ddots & \vdots \\
0 & 1 & 0 & \ddots & 0 \\
\vdots & \ddots & \ddots & \ddots & 1 \\
0 & \ldots & 0 & 1 & 0
\end{array}\right) .
$$

Note that the $k$ th eigenvalue of $\mathbf{M}_{n}\left(-b_{2}\right)$ is greater than or equal to $\tilde{\lambda}_{k}$, since $\mathbf{M}_{n}\left(-b_{2}\right) \geq \mathbf{S}_{n, 2}$. Let us also note that $\mathbf{M}_{n}(0)=\mathbf{F}_{n}$. Let us denote the $k$ th eigenvalue of $\mathbf{M}_{n}(t)$ by $\lambda_{k}(t)$ and the corresponding eigenvector by $X(t)$, normalized as $\|X(t)\|=1$. Since $\mathbf{M}_{n}(t)$ is a smooth function of $t$ around 0 and $\lambda_{k}$ is a simple eigenvalue of $\mathbf{F}_{n}$, we get that $\lambda_{k}(t)$ is a simple eigenvalue of $\mathbf{M}_{n}(t), \lambda_{k}(0)=\lambda_{k}$, and $\lambda_{k}(t)$ and $X(t)$ are smooth functions of $t$ around 0 (see Theorems 9.7 and 9.8 in [14]). Let us also observe that $\mathbf{M}_{n}(t)$ is self-adjoint, $\|X(t)\|=1$ and $\mathbf{M}_{n}(t) X(t)=\lambda_{k}(t) X(t)$. Therefore the Hellmann-Feynman Theorem (Theorem 1.4.7 in [17]) implies

$$
\lambda_{k}^{\prime}(t)=\left\langle X(t), \mathbf{M}_{n}^{\prime}(t) X(t)\right\rangle=-X_{1}^{2}(t)-X_{2}^{2}(t),
$$

where $X(t)^{\top}=\left[X_{1}(t), X_{2}(t), \ldots, X_{n}(t)\right]$. Since $X(t)$ is a non-zero eigenvector of the tridiagonal matrix $\mathbf{M}_{n}(t)$, at least one of $X_{1}(t)$ and $X_{2}(t)$ is non-zero. Therefore by Eq. (4.4), there exists an open interval $I \subset \mathbb{R}$ containing 0 such that $\lambda_{k}^{\prime}(t)<0$ for $t \in I$, i.e. $\lambda_{k}(t)$ is decreasing on $I$. This implies existence of $0<t_{0}<-b_{2}$ satisfying

$$
\lambda_{k}>\lambda_{k}\left(t_{0}\right) \geq \lambda_{k}\left(-b_{2}\right) \geq \tilde{\lambda}_{k}
$$

This contradicts with our assumption that $\lambda_{k}=\tilde{\lambda}_{k}$. Therefore only the first case of the claim is true, i.e. $b_{1}=b_{2}=0$ and hence $\mathbf{S}_{n, 2}=\mathbf{F}_{n}$.

Acknowledgements The authors would like to thank Wencai Liu for introducing them this project and his constant support. This work was partially supported by NSF DMS-2015683 and DMS-2000345.

Data Availability Data sharing is not applicable to this article as no data sets were generated or analyzed during the current study.

Open Access This article is licensed under a Creative Commons Attribution 4.0 International License, which permits use, sharing, adaptation, distribution and reproduction in any medium or format, as long as you give appropriate credit to the original author(s) and the source, provide a link to the Creative Commons licence, and indicate if changes were made. The images or other third party material in this article are included in the article's Creative Commons licence, unless indicated otherwise in a credit line to the material. If material is not included in the article's Creative Commons licence and your intended use is not permitted by statutory regulation or exceeds the permitted use, you will need to obtain permission directly from the copyright holder. To view a copy of this licence, visit http://creativecommons.org/licenses/by/4.0/. 


\section{References}

1. Ambarzumian, V.: Über eine frage der eigenwerttheorie. Zeitschrift für Physik 53, 690-695 (1929). (in German)

2. Bebiano, N., da Providência, J.: Inverse problems for pseudo-Jacobi matrices: existence and uniqueness results. Inverse Prob. 27(2), 025005 (2011)

3. Clark, S., Gesztesy, F., Renger, W.: Trace formulas and Borg-type theorems for matrix-valued Jacobi and Dirac finite difference operators. J. Differ. Equ. 219(1), 144-182 (2005). (MR2181033)

4. del Rio, R., Kudryavtsev, M.: Inverse problems for Jacobi operators: I. Interior mass-spring perturbations in finite systems. Inverse Prob. 28(5), 055007 (2012)

5. del Rio, R., Kudryavtsev, M., Silva, L.O.: Inverse problems for Jacobi operators III: Massspring perturbations of semi-infinite systems. Inverse Probl. Imag. 6(4), 599-621 (2012)

6. del Rio, R., Kudryavtsev, M., Silva, L.O.: Inverse problems for Jacobi operators IV: interior mass-spring perturbations of semiinfinite systems. Inver. Prob. 33(5), 055014 (2017)

7. Derevyagin, M.: Borg-type theorems for generalized Jacobi matrices and trace formulas. Methods Funct. Anal. Topol. 12(3), 220-233 (2006)

8. Gesztesy, F.: Inverse spectral theory as influenced by Barry Simon, Spectral theory and mathematical physics: a Festschrift in honor of Barry Simon's 60th birthday, (2007), pp. 741-820. MR2307754

9. Gesztesy, F., Kiselev, A., Makarov, K.A.: Uniqueness results for matrix-valued Schrödinger, Jacobi, and Dirac-type operators. Math. Nachr. 239/240, 103-145 (2002)

10. Gesztesy, F., Simon, B.: m-functions and inverse spectral analysis for finite and semi-infinite Jacobi matrices. J. Anal. Math. 73, 267-297 (1997)

11. Hatinoğlu, B.: Inverse problems for Jacobi operators with mixed spectral data. J. Differ. Equ. Appl. 27(1), 81-101 (2021)

12. Hatinoğlu, B.: Mixed data in inverse spectral problems for the Schrödinger operators. J. Spectr. Theory 11(1), 281-322 (2021)

13. Horváth, M.: Inverse spectral problems and closed exponential systems. Ann. Math. (2) 162(2), 885918 (2005)

14. Lax, P.D.: Linear algebra and its applications. Wiley, Interscience Series of Texts (2007)

15. Makarov, N., Poltoratski, A.: Two-spectra theorem with uncertainty. J. Spectr. Theory 9(4), 1249-1285 (2019)

16. Silva, L.O., Weder, R.: On the two spectra inverse problem for semi-infinite Jacobi matrices. Math. Phys. Anal. Geom. 9(3), 263-290 (2006)

17. Simon, B.: A Comprehensive Course in Analysis, Part 4: Operator Theory. American Mathematical Society, Providence (2015)

18. Teschl, G.: Trace formulas and inverse spectral theory for Jacobi operators. Comm. Math. Phys. 196(1), 175-202 (1998)

19. Teschl, G.: Jacobi Operators and Completely Integrable Nonlinear Lattices, Mathematical Surveys and Monographs, vol. 72. American Mathematical Society, Providence, RI (2000)

20. Teschl, G., Unterkofler, K.: Spectral theory as influenced by Fritz Gesztesy, Spectral analysis, differential equations and mathematical physics: a festschrift in honor of Fritz Gesztesy's 60th birthday, (2013), pp. 341-363. MR3087914

Publisher's Note Springer Nature remains neutral with regard to jurisdictional claims in published maps and institutional affiliations. 